\title{
Sleep-time BP: prognostic marker of type 2 diabetes and therapeutic target for prevention
}

\author{
Ramón C. Hermida $^{1}$ • Diana E. Ayala ${ }^{1}$ • Artemio Mojón ${ }^{1}$ - José R. Fernández ${ }^{1}$
}

Received: 27 April 2015 / Accepted: 19 August 2015 / Published online: 23 September 2015

(C) Springer-Verlag Berlin Heidelberg 2015

\begin{abstract}
Aims/hypothesis We investigated the prognostic value of clinic and ambulatory BP (ABP) to predict new-onset diabetes and whether risk reduction is related to the progressive decrease of clinic BP or awake or asleep ABP.

Methods We prospectively evaluated 2,656 individuals without diabetes, 1,292 men and 1,364 women, 50.6 \pm 14.3 years of age, with baseline BP ranging from normotension to hypertension according to ABP criteria. At baseline and annually (more frequently if hypertension treatment was adjusted based on ABP) thereafter, ABP and physical activity (wrist actigraphy) were simultaneously monitored for $48 \mathrm{~h}$ to accurately derive the awake and asleep BP means.

Results During a 5.9-year median follow-up, 190 participants developed type 2 diabetes. The asleep systolic ABP mean was the most significant predictor of new-onset diabetes in a Cox proportional-hazard model adjusted for age, waist circumference, glucose, chronic kidney disease (CKD) and hypertension treatment. Daytime clinic BP and awake or $48 \mathrm{~h} \mathrm{ABP}$ mean had no predictive value when corrected by the asleep ABP mean. Analyses of BP changes during follow-up revealed a $30 \%$ reduction in the risk of new-onset diabetes per 1-SD decrease in asleep systolic ABP mean, independent of changes in clinic $\mathrm{BP}$ or awake or $48 \mathrm{~h} \mathrm{ABP}$ means.
\end{abstract}

Electronic supplementary material The online version of this article (doi:10.1007/s00125-015-3748-8) contains peer-reviewed but unedited supplementary material, which is available to authorised users.

Ramón C. Hermida

rhermida@uvigo.es

1 Bioengineering and Chronobiology Laboratories, E.I.

Telecomunicación, University of Vigo, Campus Universitario, Vigo, Pontevedra 36310, Spain
Conclusions/interpretation Sleep-time BP is a highly significant independent prognostic marker for new-onset diabetes. Alteration in sleep-time BP regulation seems to precede, rather than follow, the development of new-onset diabetes. Most important, lowering asleep BP, a novel therapeutic target requiring $\mathrm{ABP}$ evaluation, could be a significant method for reducing new-onset diabetes risk.

Keywords Ambulatory blood pressure $\cdot$ New-onset diabetes . Sleep-time blood pressure

\begin{tabular}{ll}
\multicolumn{2}{l}{ Abbreviations } \\
ABP & Ambulatory BP \\
ABPM & Ambulatory BP monitoring \\
CKD & Chronic kidney disease \\
CKD-EPI & CKD Epidemiology Collaboration \\
CVD & Cardiovascular disease \\
DBP & Diastolic BP \\
MAPEC & Monitorización Ambulatoria para \\
& Predicción de Eventos Cardiovasculares \\
PP & Pulse pressure \\
SBP & Systolic BP
\end{tabular}

\section{Introduction}

Specific features of the daily BP pattern determined by ambulatory $\mathrm{BP}(\mathrm{ABP})$ monitoring $(\mathrm{ABPM})$ have been explored as biomarkers or mediators of target tissue injury and triggers of and risk factors for cardiovascular disease (CVD) events [1]. Numerous ABPM studies consistently substantiate the incidence of end-organ injury, and fatal and non-fatal CVD events are significantly associated with blunted sleep-time relative BP decline (so-called non-dipper BP pattern), not only in 
hypertensive patients both without [2-6] and with diabetes [7-11], but also in normotensive individuals [12]. Furthermore, various independent prospective studies demonstrate CVD events are better predicted by the sleep-time than awake or daily ABP means [4-6, 13-18], also in diabetes [9, 11, 19]. Most important, we recently documented that, the progressive reduction of the sleep-time BP mean by proper scheduling of hypertension treatment is the most significant predictor of CVD event-free survival [5, 6, 20, 21], including patients with diabetes [11, 22].

Night-time hypertension and non-dipper BP patterning are highly prevalent in diabetes and these features have been consistently associated with the increased CVD risk of patients with diabetes [23-27]. For instance, a recent cross-sectional study involving 12,765 patients with hypertension according to current ABPM criteria - awake systolic (SBP)/diastolic (DBP) BP mean $\geq 135 / 85 \mathrm{mmHg}$ or asleep SBP/DBP mean $\geq 120 / 70 \mathrm{mmHg}[28,29]$ — that included 2,954 participants with type 2 diabetes, found the prevalence of non-dipping was significantly higher in patients with than without diabetes (62.1\% vs $45.9 \% ; p<0.001)$ [27]. Furthermore, elevated sleep-time SBP mean was the major basis for the diagnosis of hypertension and/or inadequate BP control among patients with diabetes; thus, among the uncontrolled hypertensive patients with diabetes, $89.2 \%$ had sleep-time hypertension [27].

These findings lead us to propose two hypotheses that have never before been prospectively investigated: (1) features of the $24 \mathrm{~h}$ ABP pattern are predictive of new-onset diabetes risk and development; and (2) targeting potential prognostic ABPderived risk markers by therapeutic intervention modifies the risk of new-onset diabetes. The MAPEC study (Monitorización Ambulatoria para Predicción de Eventos Cardiovasculares, i.e., Ambulatory Blood Pressure Monitoring for Prediction of Cardiovascular Events) was specifically designed to prospectively investigate whether specific treatment-induced changes in the $24 \mathrm{~h} \mathrm{ABP}$ profile reduce risk of CVD, new-onset diabetes and renal events [30]. This study thus allowed us to prospectively evaluate whether reduced risk of developing new-onset diabetes might be related to the progressive decrease of clinic $\mathrm{BP}$ or $\mathrm{ABP}$ variables, including the awake, asleep and daily BP means.

\section{Methods}

Inclusion and exclusion criteria Complete details of the rationale and design of the MAPEC study are described in previous publications $[5,6,11,12,20-22,30]$. In summary, the sample consisted of Spanish individuals $\geq 18$ years of age who adhered to a routine of daytime activity and night-time sleep. Inclusion criteria required participants to be either normotensive, untreated hypertensive or resistant to treatment [31] when ingesting all their prescribed BP-lowering medications upon awakening. Exclusion criteria were pregnancy, history of drug/alcohol abuse, night/shift-work employment, acquired immunodeficiency syndrome (AIDS), type 1 diabetes, secondary hypertension, CVD disorders (unstable angina pectoris, heart failure, life-threatening arrhythmia, nephropathy and grade III-IV retinopathy), intolerance to ABPM, and inability to communicate and comply with all study requirements. This prospective single-centre study (ClinicalTrial.gov registration no. NCT00295542) was approved by the state Ethics Committee of Clinical Research. All participants gave written informed consent.

Participants and diagnostic criteria Between 2000 and 2007 , we recruited 3,612 persons fulfilling the inclusion/ exclusion criteria, with 3,344 (1,718 men/1,626 women, $52.6 \pm 14.5[$ mean $\pm \mathrm{SD}]$ years of age) providing all required information for study. The remaining 268 individuals were excluded due to inadequate ABPM sampling at baseline and non-consent for additional ABPM evaluations. At the time of recruitment, 688 patients already had a diagnosis of type 2 diabetes and, therefore, were also excluded from analyses. The final evaluated population for the hypotheses tested herein thus consisted of 2,656 individuals (644 normotensive and 2,012 hypertensive according to the ABPM criteria provided above) without diabetes, 1,292 men and 1,364 women, $50.6 \pm 14.3$ years of age. A major goal of the study was to assess the effect of treatment-time regimen of prescribed BP-lowering medications on CVD, new-onset diabetes and renal outcomes. Thus, hypertensive patients were randomised either to ingest all BP-lowering medications upon awakening or the complete daily dose of $\geq 1$ of them at bedtime and the remaining ones (if any) upon awakening (see Electronic Supplementary Material [ESM] Methods) [20-22, 30, 32].

New-onset diabetes was defined as fasting glucose $\geq 7.0 \mathrm{mmol} / \mathrm{l}$ on at least two clinical assessments $\geq 3$ months apart in participants without prior history of diabetes or glucose-lowering treatment [33]. Chronic kidney disease (CKD) was defined as either an estimated GFR $<60 \mathrm{ml} \mathrm{min}^{-1} 1.73 \mathrm{~m}^{-2}$, albuminuria (urinary albumin excretion $\geq 30 \mathrm{mg} / 24 \mathrm{~h}$ urine or albumin/creatinine ratio $\geq 3 \mathrm{mg} /$ $\mathrm{mmol}$ ), or both, on at least two occasions $\geq 3$ months apart [34]. GFR ( $\mathrm{ml} \mathrm{min}^{-1} 1.73 \mathrm{~m}^{-2}$ ) was estimated by the CKDEpidemiology Collaboration (CKD-EPI) equation [35]. Diagnosis of the metabolic syndrome was established by the National Cholesterol Education Program Adult Treatment Panel III revised definition [36]. Diagnosis of obstructive sleep apnoea (apnoea/hypopnoea index $\geq 10$ ) was corroborated by overnight in-hospital polysomnography when the participant or bed-mate reported sleepiness during daytime and loud snoring, choking, interrupted breathing and/or awakenings during night-time sleep. 
ABP, wrist activity and other assessments At inclusion and at every scheduled visit for ABPM during follow-up, the SBP and DBP of each participant were automatically measured every $20 \mathrm{~min}$ between 07:00 and 23:00 hours and every $30 \mathrm{~min}$ during the night for 48 consecutive hours with a calibrated SpaceLabs 90207 ABPM monitor (SpaceLabs Issaquah, WA, USA). A $48 \mathrm{~h}$, instead of the most common $24 \mathrm{~h}$, monitoring span was chosen to improve reproducibility of results, as accurate calculation of $\mathrm{ABP}$ characteristics (including mean $\mathrm{BP}$ values) and dipping classification depends markedly on the duration of ABPM [37]. Participants were instructed to adhere to their usual activities with minimal restrictions but to keep a similar activity-rest schedule and avoid daytime napping during the two consecutive days of ABPM. In keeping with current recommendations [29], BP series were considered invalid for analysis, and thus ABPM repeated within the same week, if $\geq 30 \%$ of the measurements were missing, if data were lacking for an interval of $>2 \mathrm{~h}$, if data were obtained when the restactivity schedule was irregular during the 2 days of monitoring, or if the night-time sleep period was $<6 \mathrm{~h}$ or $>12 \mathrm{~h}$.

All participants also wore an actigraph (Mini-MotionLogger, Ambulatory Monitoring, Ardsley, NY, USA) on the dominant wrist to record the level of physical activity at 1-min intervals during each $48 \mathrm{~h} \mathrm{ABPM}$. Actigraphy data were used to verify absence of daytime napping and to precisely define the commencement and termination of the daytime awake and night-time asleep spans of each participant using dedicated software [38] to accurately derive the respective ABP means.

Blood withdrawal from an antecubital vein was done in the clinic between 08:00 and 09:00 hours, after overnight fasting, the same week when each $48 \mathrm{~h}$ ABPM session was initiated. The patients collected their urine during the first $24 \mathrm{~h}$ of ABPM. Blood and urine samples were analysed using routine automatic techniques in the hospital laboratory. Just before commencing each $48 \mathrm{~h}$ ABPM session, the same investigator obtained six consecutive clinic BP measurements using a validated automatic oscillometric device (HEM-705IT, Omron Health Care, Vernon Hills, IL, USA) after the participant had rested in a seated position for $\geq 10 \mathrm{~min}$. Proper cuff size for clinic and ABP assessment was determined by measurement of upper arm circumference at each study visit.

Follow-up Participants underwent the same evaluation procedure described above, including conventional daytime clinic $\mathrm{BP}$ measurement, $48 \mathrm{~h} \mathrm{ABPM/wrist} \mathrm{activity} \mathrm{monitoring} \mathrm{and}$ blood/urine analysis, plus other complementary tests as ordered by physicians (e.g., electrocardiogram, funduscopic evaluation, echocardiogram, etc.), annually or more frequently (3 months after any doctor-ordered change in therapy to improve ABP control in treated patients). Investigators blinded to the treatment scheme of hypertensive patients and not involved in clinic evaluations, $\mathrm{ABP}$ measurement and statistical analyses assessed the development of new-onset diabetes, as defined above, among other outcome variables of interest. Complete clinical records of all enrolled participants, including all periodic laboratory tests performed during follow-up, were reviewed at least annually plus the year following their last ABPM.

Statistical methods ABPM profiles were edited according to conventional criteria to correct for measurement errors and outliers; SBP readings $>250$ or $<70 \mathrm{mmHg}$, DBP $>150$ or $<40 \mathrm{mmHg}$ and pulse pressure (PP, SBP minus DBP) $>150$ or $<20 \mathrm{mmHg}$ were automatically discarded. The ' $48 \mathrm{~h} \mathrm{ABP}$ mean' was calculated as the average of all valid readings obtained throughout the $48 \mathrm{~h}$ assessment span. Awake and asleep ABP means were calculated from the $48 \mathrm{~h}$ monitoring as the average of all valid readings obtained during the hours of daytime activity or night-time sleep, respectively, as differentiated by wrist actigraphy. Sleep-time relative BP decline (index of BP dipping), defined as \% decrease in mean BP during night-time sleep relative to mean $\mathrm{BP}$ during daytime activity, was calculated as: ([awake ABP mean-asleep ABP mean]/ awake $A B P$ mean $) \times 100$, incorporating all the data sampled by $48 \mathrm{~h}$ ABPM. For comparative purposes, a participant was defined as dipper if the sleep-time relative SBP decline was $\geq 10 \%$, and as non-dipper otherwise [28, 29].

The risk of new-onset diabetes was evaluated based on the: (1) baseline clinic and ABPM evaluation from every participant; and (2) changes in any tested clinic cuff and ABP variable per participant during follow-up. Demographic and clinical characteristics were compared among groups of individuals who did or did not develop new-onset diabetes by $t$ test (quantitative variables) or nonparametric $\chi^{2}$ test (proportions). The Cox proportional-hazard model, adjusted for significant confounding variables, was used to estimate HRs with $95 \% \mathrm{CI}$ for events associated with each tested potential prognostic BP variable; we standardised these HRs by calculating them for 1-SD increments for each BP variable. All demographic, anthropometric and clinical laboratory variables listed in Table 1 were tested as potential confounding variables. Adjustments were applied for fasting glucose, waist circumference, age, hypertension treatment-time and CKD diagnosis, as these influential factors were the only ones consistently significant in all tested Cox regression models. On the other hand, the prognostic value of $\mathrm{BP}$ changes during follow-up was evaluated by entering the change in the BP variable of interest as a time-dependent covariate in the Cox regression analysis. For survival analysis, followup was established as either the time to the confirmed diagnosis of new-onset diabetes or the time to the last clinical evaluation in individuals who did not develop new-onset diabetes.

\section{Results}

Demographic characteristics and laboratory variables in event and non-event participants During the median follow- 
Table 1 Baseline characteristics of investigated participants

\begin{tabular}{lccc}
\hline Variable & No event & Event & $\begin{array}{l}p \text { between } \\
\text { groups }\end{array}$ \\
\hline
\end{tabular}

Demographic characteristics

Patients $(n)$

Sex (\% men)

The metabolic syndrome $(\%)^{\mathrm{a}}$

Cigarette smoking (\%)

Obesity $(\%)^{\mathrm{b}}$

Obstructive sleep apnoea (\%)

Anaemia (\%)

Albuminuria (\%) ${ }^{\mathrm{d}}$

CKD (\%)

Anthropometric variables and office BP

Age (years)

Height $(\mathrm{cm})$

Weight $(\mathrm{kg})$

BMI $\left(\mathrm{kg} / \mathrm{m}^{2}\right)$

Waist $(\mathrm{cm})$

Clinic SBP $(\mathrm{mmHg})^{\mathrm{f}}$

Clinic DBP $(\mathrm{mmHg})^{\mathrm{f}}$

Clinic PP $(\mathrm{mmHg})^{\mathrm{f}}$

Clinic heart rate (beats/min) ${ }^{\mathrm{f}}$

Clinical laboratory test values

Glucose (mmol/l)

Creatinine $(\mu \mathrm{mol} / \mathrm{l})$

Uric acid ( $\mu \mathrm{mol} / \mathrm{l})$

Total cholesterol (mmol/l)

Triacylglycerol (mmol/l)

HDL-cholesterol (mmol/l)

LDL-cholesterol (mmol/l)

Haemoglobin (g/l)

Fibrinogen $(\mu \mathrm{mol} / \mathrm{l})$

Erythrocyte sedimentation rate $(\mathrm{mm} / \mathrm{h})$

Estimated GFR

Estimated GFR $<60(\%)$

Albumin, mg/24 h urine, median (interquartile range) ABP

Duration of nocturnal rest (h)

Awake SBP mean (mmHg)

Asleep SBP mean (mmHg)

$48 \mathrm{~h} \mathrm{SBP}$ mean $(\mathrm{mmHg})$

Sleep-time relative SBP decline $(\%)^{\mathrm{g}}$

Awake DBP mean (mmHg)

Asleep DBP mean $(\mathrm{mmHg})$

$48 \mathrm{~h}$ DBP mean $(\mathrm{mmHg})$

Sleep-time relative DBP decline (\%) ${ }^{\mathrm{g}}$

Awake PP mean (mmHg)

Asleep PP mean (mmHg)

$\begin{array}{lll}2,466 & 190 & \\ 47.9 & 58.4 & 0.005 \\ 43.1 & 72.6 & <0.001 \\ 15.8 & 11.1 & 0.080 \\ 37.4 & 54.2 & <0.001 \\ 8.1 & 14.2 & 0.004 \\ 5.8 & 5.8 & 0.977 \\ 14.1 & 32.8 & <0.001 \\ 18.1 & 33.2 & <0.001\end{array}$

$50.1 \pm 14.4 \quad 57.6 \pm 11.7 \quad<0.001$

$162.8 \pm 10.0 \quad 161.5 \pm 9.4 \quad 0.090$

$77.3 \pm 14.9 \quad 82.5 \pm 16.9 \quad<0.001$

$29.1 \pm 4.8 \quad 31.5 \pm 4.9 \quad<0.001$

$94.0 \pm 12.0 \quad 100.5 \pm 12.2<0.001$

$149.1 \pm 18.3 \quad 156.4 \pm 20.6<0.001$

$86.0 \pm 11.0 \quad 88.7 \pm 10.6 \quad 0.002$

$63.1 \pm 12.7 \quad 67.7 \pm 15.8 \quad<0.001$

$74.5 \pm 12.3 \quad 73.3 \pm 12.1 \quad 0.182$

$\begin{array}{lll}5.32 \pm 0.63 & 5.93 \pm 0.64 & <0.001\end{array}$

$83.1 \pm 18.6 \quad 85.8 \pm 20.3 \quad 0.123$

$333.1 \pm 95.2 \quad 362.9 \pm 89.2<0.001$

$5.49 \pm 0.98 \quad 5.63 \pm 1.34 \quad 0.089$

$\begin{array}{lll}1.26 \pm 0.83 & 1.50 \pm 0.93 \quad<0.001\end{array}$

$\begin{array}{lll}1.26 \pm 0.40 & 1.26 \pm 0.42 & 0.837\end{array}$

$3.63 \pm 0.86 \quad 3.70 \pm 1.03 \quad 0.282$

$\begin{array}{lll}142 \pm 14 & 143 \pm 13 & 0.513\end{array}$

$\begin{array}{lll}9.29 \pm 2.26 & 9.31 \pm 2.10 & 0.938\end{array}$

$13.3 \pm 10.7 \quad 14.8 \pm 11.7 \quad 0.076$

$\begin{array}{lll}81.5 \pm 17.8 & 79.5 \pm 18.1 & 0.137\end{array}$

$\begin{array}{lll}8.9 & 11.4 & 0.274\end{array}$

$9.1(5.8-\quad 16.0(7.2-\quad<0.001$ 52.8) 16.7)

$\begin{array}{lll}8.8 \pm 1.3 & 9.2 \pm 1.6 & <0.001 \\ 130.6 \pm 13.7 & 133.8 \pm 16.9 & 0.003 \\ 116.8 \pm 14.3 & 123.7 \pm 16.9 & <0.001 \\ 126.3 \pm 13.3 & 130.5 \pm 16.2 & <0.001 \\ 10.5 \pm 6.8 & 7.3 \pm 7.8 & <0.001 \\ 81.9 \pm 10.6 & 81.0 \pm 11.2 & 0.270 \\ 68.8 \pm 10.2 & 70.8 \pm 10.3 & 0.011 \\ 77.8 \pm 10.1 & 77.7 \pm 10.4 & 0.820 \\ 15.8 \pm 7.6 & 12.3 \pm 9.0 & <0.001 \\ 48.7 \pm 9.5 & 52.8 \pm 12.5 & <0.001 \\ 48.0 \pm 9.4 & 52.9 \pm 12.9 & <0.001\end{array}$


Table 1 (continued)

\begin{tabular}{llll}
\hline Variable & No event & Event & $\begin{array}{l}p \text { between } \\
\text { groups }\end{array}$ \\
\hline 48 h PP mean (mmHg) & $48.5 \pm 9.2$ & $52.8 \pm 12.3$ & $<0.001$ \\
Sleep-time relative PP decline (\%) & $1.0 \pm 9.1$ & $-0.8 \pm 11.0$ & 0.009 \\
Non-dipper (\%) & 43.2 & 62.1 & $<0.001$ \\
\hline
\end{tabular}

Values are shown as mean $\pm \mathrm{SD}$, unless otherwise stated

${ }^{a}$ The metabolic syndrome: National Cholesterol Education Program Adult Treatment Panel III revised definition [36]

${ }^{\mathrm{b}}$ Obesity: $\mathrm{BMI} \geq 30 \mathrm{~kg} / \mathrm{m}^{2}$

${ }^{\mathrm{c}}$ Anaemia: haemoglobin $<130 \mathrm{~g} / \mathrm{l}$ in men and $<120 \mathrm{~g} / \mathrm{l}$ in women

${ }^{\mathrm{d}}$ Albuminuria: urinary albumin excretion $\geq 30 \mathrm{mg} / 24 \mathrm{~h}$ urine

${ }^{\mathrm{e}} \mathrm{CKD}$ : estimated GFR $<60 \mathrm{ml} \mathrm{min}{ }^{-1} 1.73 \mathrm{~m}^{-2}$, albuminuria, or both, on at least two occasions $\geq 3$ months apart [34]. GFR ( $\mathrm{ml} \mathrm{min}{ }^{-1} 1.73 \mathrm{~m}^{-2}$ ) was estimated using the CKD-EPI equation [35]

${ }^{\mathrm{f}}$ Values correspond to the average of six conventional BP measurements obtained for each participant at the clinic before starting $48 \mathrm{~h} \mathrm{ABPM}$

${ }^{\mathrm{g}}$ Sleep-time relative BP decline, index of BP dipping, is defined as \% decline in mean BP during night-time sleep relative to mean BP during daytime activity, and calculated as: ([awake BP mean-asleep BP mean]/awake BP mean) $\times 100$

${ }^{\mathrm{h}}$ Non-dipper: participants with sleep-time relative SBP decline $<10 \%$, using data sampled by ABPM for 48 consecutive hours

up period of 5.9 years (range 1.0-8.9 years), 190 participants developed type 2 diabetes. Event-persons were: predominantly men, of older age, and who at baseline were likely to have obstructive sleep apnoea, the metabolic syndrome, abdominal obesity and/or CKD (Table 1). In addition, at baseline they had greater clinic BP and higher glucose, uric acid, triacylglycerol, plasma fibrinogen and urinary albumin levels, but lower estimated GFR (Table 1). At baseline, the $48 \mathrm{~h}$ mean of SBP, but not of DBP, was significantly greater among eventpersons (Table 1). The largest difference between the event and non-event groups was in the asleep SBP mean. The sleeptime relative SBP decline was significantly lower $(p<0.001)$ and prevalence of non-dipping significantly higher, $62 \% \mathrm{vs}$ $43 \%$, among event-persons $(p<0.001$; Table 1$)$. All differences between groups reported in Table 1 with a $p$ value $<0.002$ were significant after correcting for multiple testing. The comparison of baseline characteristics of the investigated participants, categorised as a function of dipping status, is provided in ESM Table 1.

\section{Clinic BP and ABP as predictors of new-onset diabetes} Table 2 (left column) reports the HR of new-onset diabetes estimated by the Cox proportional-hazard model, calculated on the basis of the baseline clinic and ABP evaluations of each participant. Increased risk of new-onset diabetes was associated with elevated fasting glucose (HR 3.44, 95\% CI [2.71, 4.36], $p<0.001$, per mmol/l); larger waist circumference (1.03 $[1.02,1.04], p<0.001$, per $\mathrm{cm})$; older age $(1.02[1.01,1.03]$, $p<0.001$, per year); and presence of CKD $(1.53$ [1.13, 2.08], $p=0.007$, compared with absence of CKD). Additionally, hypertensive patients ingesting $\geq 1$ BP-lowering medications at bedtime showed a significantly lower HR of new-onset diabetes than those ingesting all medications upon awakening (0.43 [0.31, 0.61]; $p<0.001)$, as extensively described elsewhere [32].

Table 2 (left column) shows the HR of new-onset diabetes was greater with progressively higher sleep-time SBP mean and lower sleep-time relative SBP decline, i.e., more nondipper BP patterning. The asleep, but not awake, SBP mean was indeed the most significant predictor of new-onset diabetes (for each 1-SD elevation, HR 1.28, 95\% CI [1.10, 1.45] for asleep SBP, $p<0.001 ; 1.12[0.97,1.28]$ for awake SBP, $p=$ 0.121; Table 2, left column). A stepwise Cox regression analysis further indicated that, just after fasting glucose, the asleep SBP mean was the second most significant prognostic marker of new-onset diabetes. Interestingly, a greater either morning or pre-awakening BP surge, calculated as previously defined [39], was significantly associated with lower, not higher, risk of new-onset diabetes (Table 2, left column; Fig. 1a). This finding is in agreement with the highly significant association between increased sleep-time relative ABP decline and attenuated risk.

Table 2 (centre column) reports the HR of new-onset diabetes based on the clinic and ABP obtained for each participant at his/her final evaluation. The findings are similar to those described above based on the corresponding data of the baseline evaluation (Table 2, left column), thereby providing further evidence for the greater prognostic value of the asleep BP mean as compared with any other ABP characteristic. Additionally, results indicate increasing prognostic value 
Table 2 Adjusted HRs of new-onset diabetes associated with clinic BP and ABP

\begin{tabular}{|c|c|c|c|}
\hline Variable & Baseline BP evaluation $^{\mathrm{a}}$ & Final BP evaluation ${ }^{\mathrm{a}}$ & Changes in BP during follow-up ${ }^{b}$ \\
\hline \multicolumn{4}{|l|}{ SBP } \\
\hline Clinic & $1.20(1.05,1.38)^{* *}$ & $1.30(1.14,1.48)^{* * *}$ & $0.94(0.73,1.21)$ \\
\hline Awake mean & $1.12(0.97,1.28)$ & $1.18(1.04,1.34)^{* *}$ & $0.85(0.73,0.99)^{*}$ \\
\hline Asleep mean & $1.28(1.10,1.45)^{* * *}$ & $1.40(1.25,1.57)^{* * *}$ & $0.70(0.61,0.81)^{* * *}$ \\
\hline $48 \mathrm{~h}$ mean & $1.16(1.01,1.33)^{*}$ & $1.26(1.12,1.42)^{* * *}$ & $0.79(0.68,0.92)^{* *}$ \\
\hline Sleep-time relative decline ${ }^{c}$ & $0.79(0.69,0.91)^{* * *}$ & $0.70(0.62,0.80)^{* * *}$ & $0.73(0.64,0.84)^{* * *}$ \\
\hline Morning surge $^{d}$ & $0.84(0.73,0.96)^{*}$ & $1.02(0.89,1.17)$ & $0.90(0.77,1.04)$ \\
\hline Pre-awakening surge ${ }^{e}$ & $0.84(0.73,0.95)^{* *}$ & $0.92(0.80,1.05)$ & $1.06(0.91,1.24)$ \\
\hline Night-time fall ${ }^{\mathrm{f}}$ & $0.83(0.73,0.95)^{* *}$ & $0.81(0.70,0.93)^{* *}$ & $1.07(0.93,1.25)$ \\
\hline \multicolumn{4}{|l|}{ DBP } \\
\hline Clinic & $1.18(1.02,1.36)^{*}$ & $1.28(1.12,1.46)^{* * *}$ & $1.02(0.77,1.33)$ \\
\hline Awake mean & $1.05(0.90,1.22)$ & $1.21(1.06,1.39)^{* *}$ & $0.86(0.73,1.00)$ \\
\hline Asleep mean & $1.18(1.02,1.35)^{* *}$ & $1.46(1.29,1.66)^{* * *}$ & $0.72(0.62,0.83)^{* * *}$ \\
\hline $48 \mathrm{~h}$ mean & $1.09(0.93,1.25)$ & $1.29(1.14,1.48)^{* * *}$ & $0.80(0.69,0.93)^{* *}$ \\
\hline Sleep-time relative decline ${ }^{c}$ & $0.80(0.70,0.92)^{* *}$ & $0.73(0.64,0.83)^{* * *}$ & $0.78(0.69,0.90)^{* * *}$ \\
\hline Morning surge $^{\mathrm{d}}$ & $0.87(0.77,1.02)$ & $0.94(0.81,1.08)$ & $1.00(0.86,1.17)$ \\
\hline Pre-awakening surge ${ }^{e}$ & $0.80(0.69,0.93)^{* *}$ & $0.85(0.73,0.98)^{*}$ & $1.08(0.92,1.27)$ \\
\hline Night-time fall ${ }^{\mathrm{f}}$ & $0.92(0.80,1.06)$ & $0.82(0.71,0.95)^{* *}$ & $1.18(0.99,1.37)$ \\
\hline \multicolumn{4}{|l|}{ PP } \\
\hline Clinic & $1.14(0.99,1.31)$ & $1.20(1.05,1.38)^{* *}$ & $0.93(0.73,1.18)$ \\
\hline Awake mean & $1.12(0.98,1.29)$ & $1.07(0.93,1.23)$ & $0.95(0.83,1.10)$ \\
\hline Asleep mean & $1.23(1.06,1.39)^{* *}$ & $1.19(1.03,1.35)^{* *}$ & $0.85(0.75,0.96)^{*}$ \\
\hline $48 \mathrm{~h}$ mean & $1.16(1.01,1.33)^{*}$ & $1.11(0.97,1.28)$ & $0.91(0.79,1.04)$ \\
\hline Sleep-time relative decline ${ }^{c}$ & $0.87(0.77,0.99)^{*}$ & $0.83(0.72,0.95)^{* *}$ & $0.81(0.70,0.92)^{* *}$ \\
\hline Morning surge $^{\mathrm{d}}$ & $0.95(0.83,1.09)$ & $1.04(0.90,1.19)$ & $0.84(0.74,0.95)^{*}$ \\
\hline Pre-awakening surge ${ }^{\mathrm{e}}$ & $0.96(0.85,1.09)$ & $1.05(0.92,1.19)$ & $1.00(0.85,1.16)$ \\
\hline Night-time fall $^{\mathrm{f}}$ & $0.90(0.78,1.03)$ & $0.99(0.86,1.13)$ & $0.90(0.77,1.04)$ \\
\hline
\end{tabular}

${ }^{a}$ Baseline and final BP evaluation: HRs $(95 \%$ CI) calculated for each 1-SD elevation in BP either at the baseline evaluation upon recruitment (left column) or at the last available evaluation per participant (centre column). Adjustments were applied for significant influential characteristics of age, waist circumference, fasting glucose, diagnosis of $\mathrm{CKD}$ and hypertension treatment-time

${ }^{\mathrm{b}}$ Changes during follow-up: HRs $(95 \% \mathrm{CI})$ calculated for 1-SD decrease in BP and 1-SD increase in sleep-time relative BP decline, morning surge, preawakening surge, or night-time fall during follow-up, i.e., difference for any BP variable between the baseline and the last available evaluation per participant (right column). Adjustments were applied for significant influential characteristics of age, waist circumference, fasting glucose, diagnosis of $\mathrm{CKD}$, baseline $\mathrm{BP}$ and hypertension treatment-time. Change in BP was entered as a time-dependent covariate in the Cox regression models

${ }^{\mathrm{c}}$ Sleep-time relative BP decline, index of BP dipping, is defined as \% decline in BP during night-time sleep relative to mean BP during daytime activity, and calculated as: ([awake BP mean-asleep BP mean]/awake BP mean) $\times 100$

${ }^{\mathrm{d}}$ Morning BP surge was calculated as difference between average BP during first $2 \mathrm{~h}$ after wake-up time (i.e., morning BP) and hourly BP average centred on lowest BP reading recorded during night-time sleep (i.e., lowest sleep BP)

${ }^{\mathrm{e}}$ Pre-awakening BP surge was calculated as difference between average BP during first $2 \mathrm{~h}$ after wake-up time and average BP during $2 \mathrm{~h}$ just before wake-up time

${ }^{\mathrm{f}}$ Night-time fall was calculated as difference between average BP during the $2 \mathrm{~h}$ just before going to bed and hourly average centred on lowest BP reading recorded during night-time sleep

${ }^{*} p<0.05, * * p<0.01, * * * p<0.001$

of ABP during the years of follow-up. Finally, the prognostic value of asleep SBP mean for predicting new-onset diabetes was significant independent of absence/presence of hypertension treatment $(p<0.001)$ and time-of-day of treatment, i.e., for participants randomised to the awakening $(p<0.001)$ and bedtime ( $p=0.004)$ treatment groups analysed separately.
We next evaluated the potential combined contribution to the risk of new-onset diabetes of the multiple BP variables listed in Table 2. When the asleep SBP mean was jointly evaluated with either clinic SBP, awake or $48 \mathrm{~h}$ SBP means, or morning SBP surge, only the first significantly predicted new-onset diabetes (Fig. 1b). Only the sleep-time relative SBP 
$\mathbf{a}$



b

Clinic BP

Awake mean

Asleep mean

$48 \mathrm{~h}$ mean

Sleep-time rel. dec.

Morning surge

Pre-awak. surge

Sleep-time fall

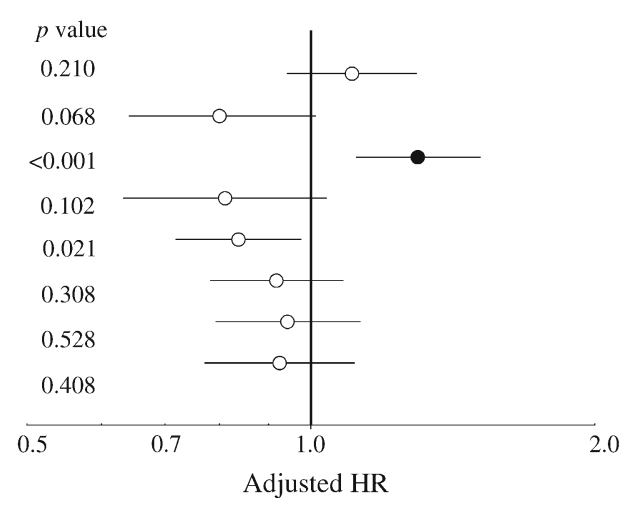

Fig. 1 Adjusted HR $(95 \% \mathrm{CI})$ of new-onset diabetes per 1-SD elevation in baseline clinic and ambulatory SBP. (a) Each tested variable evaluated separately. (b) Results adjusted by asleep SBP mean. Adjustments were applied for significant influential characteristics of age, waist circumference, fasting glucose, diagnosis of $\mathrm{CKD}$ and hypertension treatment-time. Sleep-time rel. dec., sleep-time relative decline; Pre-awak. surge, preawakening surge

decline provided additional prognostic value in an adjusted Cox regression model already including the asleep SBP mean (Fig. 1b). To further investigate the clinical relevance of the awake and asleep BP means on new-onset diabetes risk, the participants were categorised into four groups according to ABP level, i.e., normal or elevated, independent of clinic $\mathrm{BP}$, using established ABPM thresholds of $135 / 85 \mathrm{mmHg}$ for the awake SBP/DBP means and of $120 / 70 \mathrm{mmHg}$ for the asleep SBP/DBP means [28, 29]. Figure 2a, which shows the results of this analysis, indicates: (1) equivalent adjusted HR for new-onset diabetes of participants with normal asleep BP whether the awake BP mean is normal or elevated ( $p=0.374)$; (2) equivalent HR in hypertensive patients with elevated asleep BP, independent of awake BP mean $(p=0.452)$; and (3) higher adjusted HR of new-onset diabetes in participants with elevated asleep BP mean than those with normal asleep $\mathrm{BP}$, whether the awake BP mean is below or above 135/ $85 \mathrm{mmHg}$ (always $p<0.001$ ).

Exploration of the combined contribution to the risk of new-onset diabetes of multiple BP variables revealed no predictive value of clinic SBP when corrected by the asleep SBP mean (HR 1.10 [0.94, 1.29], $p=0.210$; Fig. 1b). Indeed, when



b

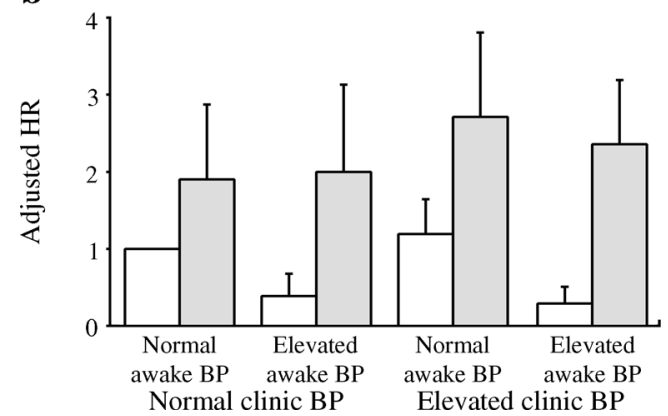

Fig. 2 (a) Adjusted HR $(95 \% \mathrm{CI})$ of new-onset diabetes as a function of awake and asleep SBP/DBP means; white bars denote normal awake BP mean; grey bars denote elevated awake BP mean. (b) Adjusted HR $(95 \%$ $\mathrm{CI}$ ) of new-onset diabetes as a function of clinic BP and awake plus asleep SBP/DBP means; white bars denote normal asleep BP mean; grey bars denote elevated asleep BP mean. Awake SBP/DBP mean was considered normal if $<135 / 85 \mathrm{mmHg}$ and elevated otherwise. Asleep SBP/DBP mean was considered normal if $<120 / 70 \mathrm{mmHg}$ and elevated otherwise. Clinic SBP/DBP was considered normal if $<140 / 90 \mathrm{mmHg}$ and elevated otherwise. Adjustments were applied for the same variables as in Fig. 1

each of the four groups of participants categorised by awake and asleep BP means (Fig. 2a) were further categorised according to either normal or elevated clinic BP (using the currently accepted 140/90 $\mathrm{mmHg}$ thresholds), new-onset diabetes risk was significantly higher in all of the four groups classified by elevated asleep ABP mean - regardless of whether clinic BP or awake ABP mean was normal or elevated - than in all of the other four groups of patients classified by normal sleep-time ABP mean (Fig. 2b).

Changes in clinic and ABP during follow-up as predictors of new-onset diabetes Table 2 (right column) presents the results of the time-dependent Cox regression analysis (adjusted by the significant confounders of age, waist circumference, fasting glucose, CKD diagnosis, baseline BP and hypertension treatment-time) of changes in BP during follow-up, i.e., difference for any tested BP variable between the values obtained upon recruitment and at the final evaluation per participant. The progressive decrease in asleep SBP mean and increase in sleep-time relative SBP decline towards a more dipper BP patterning were associated with significantly increased event-free survival, i.e., absence of new-onset diabetes (Fig. 3a). Changes in morning BP surge, preawakening BP surge and night-time BP fall during follow- 
a

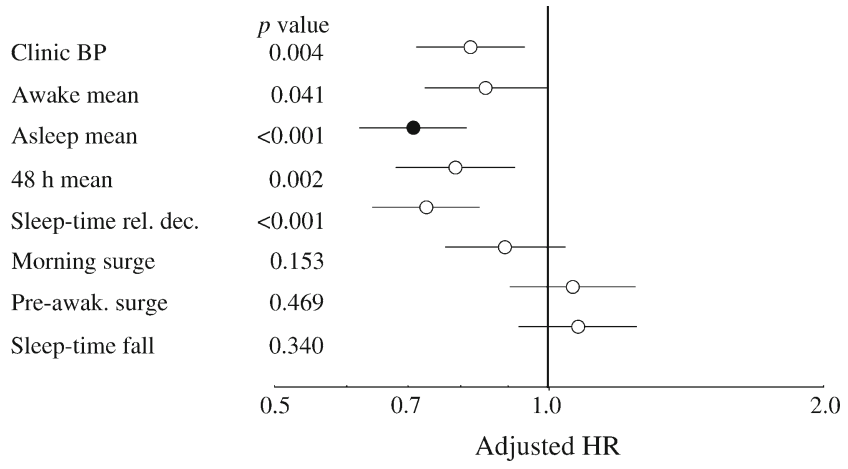

b

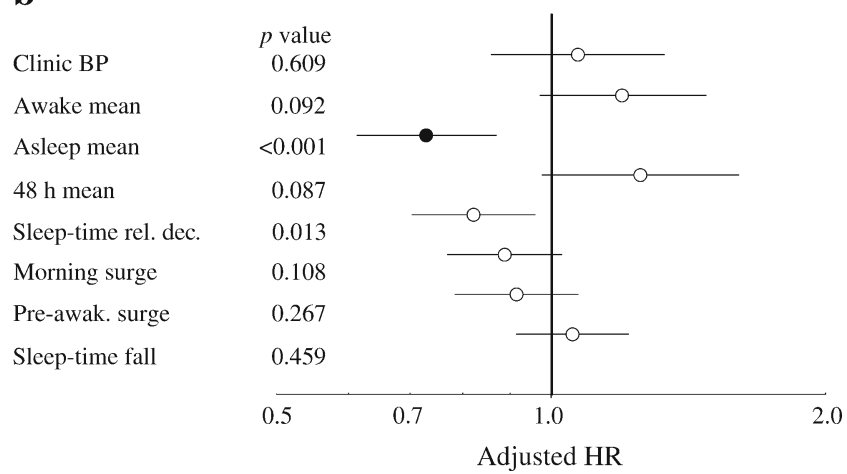

Fig. 3 Adjusted HR (95\% CI) of new-onset diabetes for each 1-SD change during follow-up in clinic and ambulatory SBP from baseline. (a) Each tested variable evaluated separately. (b) Results adjusted by asleep SBP mean. Adjustments were applied for significant influential characteristics of age, waist circumference, fasting glucose, diagnosis of $\mathrm{CKD}$, baseline BP and hypertension treatment-time. Sleep-time rel. dec., sleep-time relative decline; Pre-awak. surge, pre-awakening surge up were mainly not significantly associated with reduced/ increased risk (Table 2, right column). When the changes in clinic and ABPM-derived SBP variables were adjusted by changes in asleep SBP mean during follow-up, only the decrease in asleep SBP mean and increase in sleep-time SBP decline remained significantly associated with increased survival (Fig. 3b).

Figure 4 shows, for the studied population divided into quintiles, the relationship between new-onset diabetes risk and achieved clinic BP and ABP at final evaluation. For the clinic and awake SBP mean, the adjusted HR was only slightly higher in the last compared with the first four quintiles. On the contrary, there was a highly significant decrease in newonset diabetes risk with progressively lower achieved asleep SBP mean throughout all the quintiles (Fig. 4c). Additionally, the adjusted HR of new-onset diabetes also increased significantly when the achieved sleep-time SBP decline was $<12 \%$ (Fig. 4d).

\section{Discussion}

The MAPEC study is the first to assess prospectively, in a relatively large cohort of persons with baseline ABP ranging from normotension to hypertension, the prognostic value for new-onset diabetes not just of clinic BP and ABP variables upon recruitment, but also of their changes during a follow-up of sufficient duration by systematic periodic (at least annual) evaluation, using highly reproducible $48 \mathrm{~h}$ ABPM and wrist actigraphy. Results document that daytime conventional clinic
Fig. 4 Adjusted HR of newonset diabetes as a function of achieved clinic SBP (a) and ABPM-derived awake SBP mean (b), asleep SBP mean (c) and sleep-time relative SBP decline (d). Studied population was divided into five classes of equal size (quintiles). Numbers inside each bar provide the mean (SD) of the studied variable within each class. Adjustments were applied for the same variables as in Fig. 1. ${ }^{*} p<0.05,{ }^{* *} p<0.01$, $* * * p<0.001$, as indicated a

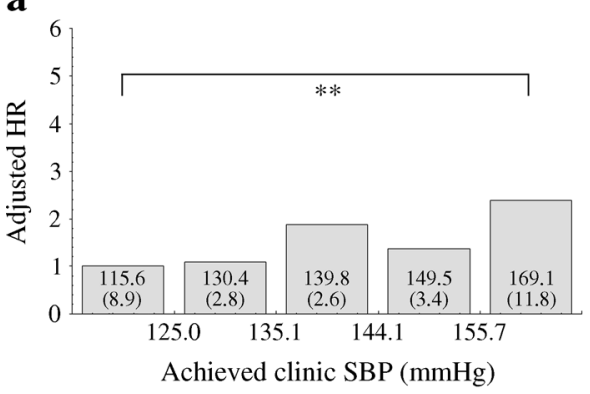

c

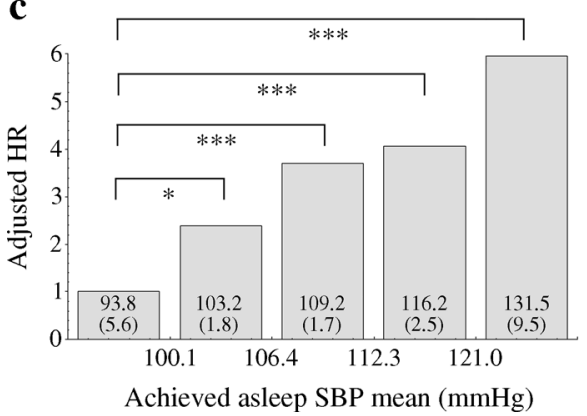

b



d

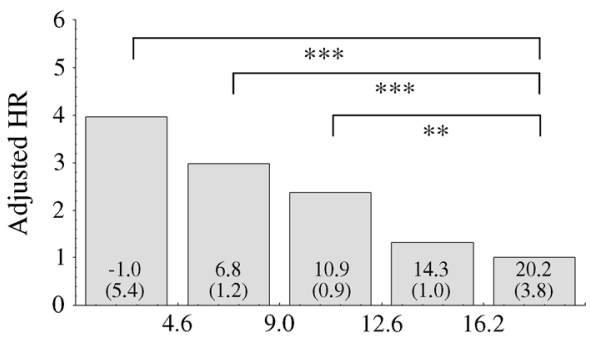

Achieved sleep-time relative SBP decline (\%) 
BP measurement is neither a significant predictor nor a proper therapeutic target for reducing the risk of new-onset diabetes (Figs 2, 3). Analyses based on the $48 \mathrm{~h}$ ABP profile obtained from each participant both at baseline, as customary in all previous studies entailing ABPM for predicting CVD events, as well as at the final evaluation, indicate that the asleep SBP mean is the most significant predictor of new-onset diabetes among the different evaluated individual ABP variables (Table 2), both individually and jointly when combined with other ABP potential prognostic markers in the best possible Cox proportional-hazard model (Fig. 1). In fact, only the sleep-time relative SBP decline added significant prognostic value to the model that already included the asleep SBP mean and corrected for relevant confounding variables. Moreover, when the asleep SBP mean was adjusted for clinic SBP or either the awake or $48 \mathrm{~h}$ SBP means, only the former was a significant and independent predictor of outcome. These results suggest the sleep-time relative SBP decline, as a continuous variable, and not just the dipping classification usually based on an arbitrary $10 \%$ threshold value, might be used to assess risk for future new-onset diabetes.

Most important from the perspective of disease prevention, evaluation of changes in the ABP variables during follow-up documents that the progressive decrease in the asleep SBP mean is significantly associated with reduced risk of newonset diabetes. The relationship between decreasing asleep ABP mean and attenuated risk of developing new-onset diabetes during follow-up is significant at all levels of the baseline asleep ABP mean, ranging from normotension to hypertension, suggesting benefit even below the current 120/ $70 \mathrm{mmHg}$ thresholds for the asleep SBP/DBP means currently used for making the diagnosis of sleep-time hypertension [28, 29]. Indeed, analysis of the adjusted HR for new-onset diabetes in terms of the achieved asleep SBP mean at the last available evaluation per participant reveals significant risk reduction even when the sleep-time SBP mean is $<100 \mathrm{mmHg}$ (Fig. 4c). All together, these novel results indicate not only that the asleep SBP mean is the most significant and independent prognostic marker for development of newonset diabetes, but they also document that decreasing the asleep SBP mean is significantly protective, independent of the other conventionally considered variables linked with increased risk of new-onset diabetes, such as elevated fasting glucose, older age, larger waist circumference and CKD diagnosis.

The findings from this prospective study thus suggest the asleep SBP mean might be a novel therapeutic target for reduction of the risk of new-onset diabetes. Along these lines, a relatively large number of previous prospective trials [40, 41] have detected meaningful morning-evening, treatment-time differences in sleep-time BP-lowering efficacy of different classes of hypertension medications and their combinations. In particular, the bedtime, in comparison with awakening, ingestion schedule of angiotensin receptor blocker and ACE inhibitor medications results in greater therapeutic effect on asleep BP independent of terminal half-life [40-42]. Accordingly, blocking the renin-angiotensin-aldosterone system by bedtime treatment seems to be an efficient means of reducing the asleep BP mean and increasing the sleep-time relative BP decline towards the normal dipper pattern. Despite the multiple factors influencing the mostly predictable daily BP variation and sleep-BP level, including rest-activity associated changes in behaviour, day-night cycles of environmental phenomena, and innate circadian $(\sim 24 \mathrm{~h})$ rhythms in neuroendocrine, endothelial and haemodynamic variables [1, $43,44]$, the impact of hypertension treatment-time on asleep $\mathrm{BP}$ regulation might thus be clinically relevant not only for CVD risk reduction - as previously documented [20-22], but also for preventing new-onset diabetes [32].

Our study has some potential limitations. First, compared with other larger multicentre clinical trials on hypertensive patients entailing clinic BP only, the sample size of the MAPEC study might seem small. Nonetheless, it is considerably greater than most other published trials on the prognostic value of ABPM [2, 3], our study being the only one yet to evaluate prospectively the prognostic value of ABP for predicting new-onset diabetes. Second, the reported findings, showing an association between elevated sleep-time BP and risk of new-onset diabetes, require validation and extrapolation to other ethnic groups. The design of the MAPEC study also has several strengths, mainly being the first to provide results that are based on systematic periodic multiple evaluations by ABPM throughout the median 5.9 years of follow-up. This so-far unique approach allowed determination of the impact of specific changes in the characteristics of the $24 \mathrm{~h} \mathrm{BP}$ profile that occurred during follow-up on the risk of developing new-onset diabetes. Further strengths of our study are use of: (1) $48 \mathrm{~h}$, instead of the most common $24 \mathrm{~h} \mathrm{ABPM} \mathrm{sam-}$ pling, to increase reproducibility of the BP findings [37]; and (2) wrist actigraphy to precisely and individually determine the beginning and end of the activity and sleep spans for each participant to enable accurate calculation of the awake and asleep ABP means.

In conclusion, according to this prospective evaluation, the sleep-time SBP mean, but not the daytime clinic BP values or the awake ABP mean, is a highly significant and independent prognostic marker of new-onset diabetes. Alteration of sleeptime BP regulation, highly frequent in diabetes, seems to precede and predict, rather than follow, development of newonset diabetes. These findings support ABPM to accurately detect abnormal sleep-time BP and, beyond its documented merit for assessing CVD risk [4-6, 9, 11-19], evaluate risk of progression to new-onset diabetes. More important, therapeutic targeting of abnormalities of the asleep SBP mean and sleep-time relative SBP decline, most effectively achieved by bedtime ingestion of the full daily dose of conventional 
hypertension medications [40-42], is associated with significantly lower risk for developing new-onset diabetes.

Duality of interest The authors declare that there is no duality of interest associated with this manuscript.

Contribution statement $\mathrm{RCH}$ and DEA contributed to every aspect of this article, including conception and design of the study, acquisition and interpretation of the data, and drafting the manuscript. AM and JRF contributed to study design, discussion, statistical analyses and revision of the manuscript. All listed authors approved the final version of the manuscript. RCH is the guarantor of this work. The authors report no potential conflicts of interest relevant to this article.

Funding This independent investigator-promoted research was supported by unrestricted grants from Ministerio de Ciencia e Innovación (SAF2006-6254-FEDER; SAF2009-7028-FEDER); Xunta de Galicia (PGIDIT03-PXIB-32201PR; INCITE07-PXI-322003ES; INCITE08E1R-322063ES; INCITE09-E2R-322099ES; 09CSA018322PR); and Vicerrectorado de Investigación, University of Vigo.

\section{References}

1. Portaluppi F, Tiseo R, Smolensky MH, Hermida RC, Ayala DE, Fabbian F (2012) Circadian rhythms and cardiovascular health. Sleep Med Rev 16:151-166

2. Verdecchia P, Porcellati C, Schillaci G et al (1994) Ambulatory blood pressure: an independent predictor of prognosis in essential hypertension. Hypertension 24:793-801

3. Ohkubo T, Hozawa A, Yamaguchi J et al (2002) Prognostic significance of the nocturnal decline in blood pressure in individuals with and without high 24-h blood pressure: the Ohasama study. J Hypertens 20:2183-2189

4. Dolan E, Stanton A, Thijs L et al (2005) Superiority of ambulatory over clinic blood pressure measurement in predicting mortality: the Dublin outcome study. Hypertension 46:156-161

5. Hermida RC, Ayala DE, Mojón A, Fernández JR (2011) Decreasing sleep-time blood pressure determined by ambulatory monitoring reduces cardiovascular risk. J Am Coll Cardiol 58:1165-1173

6. Hermida RC, Ayala DE, Fernández JR, Mojón A (2013) Sleep-time blood pressure: prognostic value and relevance as a therapeutic target for cardiovascular risk reduction. Chronobiol Int 30:68-86

7. Nakano S, Fukuda M, Hotta F et al (1998) Reversed circadian blood pressure rhythm is associated with occurrences of both fatal and nonfatal events in NIDDM subjects. Diabetes 47:1501-1506

8. Sturrock NDC, George E, Pound N, Stevenson J, Peck GM, Sowter $\mathrm{H}$ (2000) Non-dipping circadian blood pressure and renal impairment are associated with increased mortality in diabetes mellitus. Diabet Med 17:360-364

9. Astrup AS, Nielsen FS, Rossing P et al (2007) Predictors of mortality in patients with type 2 diabetes with or without diabetic nephropathy: a follow-up study. J Hypertens 25:2479-2485

10. Eguchi K, Pickering TG, Hoshide S et al (2008) Ambulatory blood pressure is a better marker than clinic blood pressure in predicting cardiovascular events in patients with/without type 2 diabetes. Am J Hypertens 21:443-450

11. Hermida RC, Ayala DE, Mojón A, Fernández JR (2012) Sleep-time blood pressure as a therapeutic target for cardiovascular risk reduction in type 2 diabetes. Am J Hypertens 25:325-334
12. Hermida RC, Ayala DE, Mojón A, Fernández JR (2013) Blunted sleep-time relative blood pressure decline increases cardiovascular risk independent of blood pressure level - the "normotensive nondipper" paradox. Chronobiol Int 30:87-98

13. Kikuya M, Ohkubo T, Asayama K et al (2005) Ambulatory blood pressure and 10-year risk of cardiovascular and noncardiovascular mortality. Ohasama Study Hypertens 45:240-245

14. Ben-Dov IZ, Kark JD, Ben-Ishay D, Mekler J, Ben-Arie L, Bursztyn M (2007) Predictors of all-cause mortality in clinical ambulatory monitoring. Unique aspects of blood pressure during sleep. Hypertension 49:1235-1241

15. Fagard RH, Celis H, Thijs L et al (2008) Daytime and nighttime blood pressure as predictors of death and cause-specific cardiovascular events in hypertension. Hypertension 51:55-61

16. Minutolo R, Agarwal R, Borrelli S et al (2011) Prognostic role of ambulatory blood pressure measurement in patients with nondialysis chronic kidney disease. Arch Intern Med 171:1090-1098

17. Hermida RC, Ayala DE, Mojón A, Smolensky MH, Portaluppi F, Fernández JR (2014) Sleep-time ambulatory blood pressure as a novel therapeutic target for cardiovascular risk reduction. J Hum Hypertens 28:567-574

18. The ABC-H Investigators, Roush GC, Fagard RH et al (2014) Prognostic impact from clinic, daytime, and nighttime systolic blood pressure in 9 cohorts on 13,844 patients with hypertension. J Hypertens 32:2332-2340

19. Bouhanick B, Bongard V, Amar J, Bousquel S, Chamontin B (2008) Prognostic value of nocturnal blood pressure and reversedipping status on the occurrence of cardiovascular events in hypertensive diabetic patients. Diabetes Metab 34:560-567

20. Hermida RC, Ayala DE, Mojón A, Fernández JR (2011) Bedtime dosing of antihypertensive medications reduces cardiovascular risk in CKD. J Am Soc Nephrol 22:2313-2321

21. Ayala DE, Hermida RC, Mojón A, Fernández JR (2013) Cardiovascular risk of resistant hypertension: dependence on treatment-time regimen of blood pressure-lowering medications. Chronobiol Int 30:340-352

22. Hermida RC, Ayala DE, Mojón A, Fernández JR (2011) Influence of time of day of blood pressure-lowering treatment on cardiovascular risk in hypertensive patients with type 2 diabetes. Diabetes Care 34:1270-1276

23. Fogari R, Zoppi A, Malamani GD, Lazzari P, Destro M, Corradi L (1993) Ambulatory blood pressure monitoring in normotensive and hypertensive type 2 diabetes. Prevalence of impaired diurnal blood pressure patterns. Am J Hypertens 6:1-7

24. Cuspidi C, Meani S, Lonati L et al (2006) Short-term reproducibility of a non-dipping pattern in type 2 diabetic hypertensive patients. J Hypertens 24:647-653

25. Afsar B, Sezer S, Elsurer R, Ozdemir FN (2007) Is HOMA index a predictor of nocturnal nondipping in hypertensives with newly diagnosed type 2 diabetes mellitus? Blood Press Monit 12:133-139

26. Pistrosch F, Reissmann E, Wildbrett J, Koehler C, Hanefeld M (2007) Relationship between diurnal blood pressure variation and diurnal blood glucose levels in type 2 diabetic patients. Am J Hypertens 20:541-545

27. Ayala DE, Moyá A, Crespo JJ et al (2013) Circadian pattern of ambulatory blood pressure in hypertensive patients with and without type 2 diabetes. Chronobiol Int 30:99-115

28. Mancia G, Fagard R, Narkiewicz K et al (2013) 2013 ESH/ESC Guidelines for the management of arterial hypertension: the Task Force for the management of arterial hypertension of the European Society of Hypertension (ESH) and of the European Society of Cardiology (ESC). J Hypertens 31:1281-1357

29. Hermida RC, Smolensky MH, Ayala DE et al (2013) 2013 ambulatory blood pressure monitoring recommendations for the diagnosis of adult hypertension, assessment of cardiovascular and other 
hypertension-associated risk, and attainment of therapeutic goals. Joint recommendations from the International Society for Chronobiology (ISC), American Association of Medical Chronobiology and Chronotherapeutics (AAMCC), Spanish Society of Applied Chronobiology, Chronotherapy, and Vascular Risk (SECAC), Spanish Society of Atherosclerosis (SEA), and Romanian Society of Internal Medicine (RSIM). Chronobiol Int 30:355-410

30. Hermida RC, Ayala DE, Mojón A, Fernández JR (2010) Influence of circadian time of hypertension treatment on cardiovascular risk: results of the MAPEC study. Chronobiol Int 27:1629-1651

31. Calhoun DA, Jones D, Textor S et al (2008) Resistant hypertension: diagnosis, evaluation, and treatment. A scientific statement from the American Heart Association Professional Education Committee of the Council for High Blood Pressure Research. Hypertension 51:1403-1419

32. Hermida RC, Ayala DE, Mojón A, Fernández JR (2015) Bedtime ingestion of hypertension medications reduces the risk of new-onset type 2 diabetes: a randomised controlled trial. Diabetologia. doi:10.1007/s00125-015-3749-7

33. American Diabetes Association (2013) Standards of Medical Care in Diabetes - 2013. Diabetes Care 36(Suppl 1):S11-S66

34. National Kidney Foundation (2002) K/DOQI clinical practice guidelines on chronic kidney disease: evaluation, classification and stratification. Am J Kidney Dis 39(2 Suppl 1):S1-S266

35. Levey AS, Stevens LA, Schmid CH et al (2009) A new equation to estimate glomerular filtration rate. Ann Intern Med 150:604-612

36. Grundy SM, Cleeman JI, Daniels SR et al (2005) Diagnosis and management of the metabolic syndrome. An American Heart Association/National Heart, Lung, and Blood Institute Scientific Statement. Circulation 112:2735-2752
37. Hermida RC, Ayala DE, Fontao MJ, Mojón A, Fernández JR (2013) Ambulatory blood pressure monitoring: importance of sampling rate and duration -48 versus 24 hours - on the accurate assessment of cardiovascular risk. Chronobiol Int 30:55-67

38. Crespo C, Fernández JR, Aboy M, Mojón A (2013) Clinical application of a novel automatic algorithm for actigraphy-based activity and rest period identification to accurately determine awake and asleep ambulatory blood pressure parameters and cardiovascular risk. Chronobiol Int 30:43-54

39. Kario K, Pickering TG, Umeda Y et al (2003) Morning surge in blood pressure as a predictor of silent and clinical cerebrovascular disease in elderly hypertensives: a prospective study. Circulation 107:1401-1406

40. Smolensky MH, Hermida RC, Ayala DE, Tiseo R, Portaluppi F (2010) Administration-time-dependent effect of blood pressurelowering medications: basis for the chronotherapy of hypertension. Blood Press Monit 15:173-180

41. Hermida RC, Ayala DE, Fernández JR et al (2013) Administrationtime-differences in effects of hypertension medications on ambulatory blood pressure regulation. Chronobiol Int 30:280-314

42. Hermida RC, Ayala DE, Fernández JR, Portaluppi F, Fabbian F, Smolensky MH (2011) Circadian rhythms in blood pressure regulation and optimization of hypertension treatment with ACE inhibitor and ARB medications. Am J Hypertens 24:383-391

43. Hermida RC, Ayala DE, Portaluppi F (2007) Circadian variation of blood pressure: the basis for the chronotherapy of hypertension. Adv Drug Deliv Rev 59:904-922

44. Fabbian F, Smolensky MH, Tiseo R, Pala M, Manfredini R, Portaluppi F (2013) Dipper and non-dipper blood pressure 24hour patterns: circadian rhythm-dependent physiologic and pathophysiologic mechanisms. Chronobiol Int 30:17-30 